\title{
New impact factor and Belgian Brain Congress
}

\author{
Jean Schoenen
}

Published online: 4 August 2012

(C) Belgian Neurological Society 2012

\section{Dear Readers,}

The impact factor is just one measure of the health of a scientific journal. It is nevertheless the yearly conundrum in June. And this year the information for the Acta Neurologica Belgica is extremely encouraging: our impact factor has risen from 0.416 in 2010 to 0.535 in 2011. This $29 \%$ increase is even more impressive if one considers that it is the result of citations of articles published before we moved in January 2012 to our new publisher Springer and its web submission system Editorial Manager. These changes by themselves should boost citations and visibility, and thus the Impact Factor, so that the future for our journal looks rather bright. Whatever this future may be, however, the quality of the ANB depends solely on your enduring and invaluable commitment, that of our readership, of our authors, and not the least, that of our reviewers and my co-editors. I take this opportunity to express my sincere thanks to all of you.

This 3rd ANB issue of 2012 covers again a vast spectrum of neurological and neurosurgical disorders spanning from transient global amnesia and neoplastic, paraneoplastic, degenerative or infectious CNS disorders to PNS diseases via trigeminal neuralgia and migraine. For the sake of an upcoming event, however, I would like to pinpoint three articles. The 1st by Mormont et al. provides most interesting informations about the disclosure of diagnosis in Belgian Alzheimer patients and their caregivers. The 2 nd by Konukoglu et al. deals with serum growth factors as biomarkers for dementia and Alzheimer's disease severity. The 3rd by Etgen et al. reports cognitive impairment as the first clinical manifestation in a case of late onset relapsing-remitting multiple sclerosis. Why emphasize these articles and what is their common denominator? The explanation is straightforward: "Cognition and Memory in Health and Disease" is precisely the topic of the next Belgian Brain Congress on October 27th 2012 in Liège. The Belgian Neurological Society is the founding stakeholder in the Belgian Brain Council, the organizer of the biennial Belgian Brain Congress. As usual, this congress is unique by its multidisciplinarity. Cognitive and memory functions will be dealt with along multiple complimentary paths going from basic to clinical sciences, from their impairment in various neurological disorders including multiple sclerosis to biomarkers for the dementias and medico-social aspects of patient care. The articles cited above touch upon various aspects that will be presented in detail during the BBC2012, the program of which can be found at the end of this issue. Have a look and join us in Liège.

Jean Schoenen

Editor-in-Chief

July 2012
J. Schoenen $(\square)$

University of Liège, Liège, Belgium

e-mail: jschoenen@ulg.ac.be 